Rezension zu: Ulrich Riegel, Stefan Heil, Boris Kalbheim, Alexander Unser (Hg.): Understanding Religion. Empirical Perspecitves in Practical Theology - Essays in Honour of Hans-Georg Ziebertz (Research on Religious and Spiritual Education 13), Münster, New York: Waxmann 2019

\author{
Müller, Sabrina
}

DOI: https://doi.org/10.1515/zpt-2020-0058

Posted at the Zurich Open Repository and Archive, University of Zurich ZORA URL: https://doi.org/10.5167/uzh-203293

Journal Article

Published Version

Originally published at:

Müller, Sabrina (2020). Rezension zu: Ulrich Riegel, Stefan Heil, Boris Kalbheim, Alexander Unser (Hg.): Understanding Religion. Empirical Perspecitves in Practical Theology - Essays in Honour of Hans-Georg Ziebertz (Research on Religious and Spiritual Education 13), Münster, New York: Waxmann 2019. Zeitschrift für Pädagogik und Theologie, 72(4):527-530.

DOI: https://doi.org/10.1515/zpt-2020-0058 


\section{Ulrich Riegel, Stefan Heil, Boris Kalbheim, Alexander Unser (Hg.): Under- standing Religion. Empirical Perspecitves in Practical Theology - Essays in Honour of Hans-Georg Ziebertz (Research on Religious and Spiritual Education 13), Münster, New York: Waxmann 2019, 254 S., € 34,90.}

Besprochen von: Dr. Sabrina Müller: Universität Zürich, Theologische Fakultät, Zentrum für Kirchenentwicklung, Kirchgasse 9, 8001 Zürich, Schweiz, E-Mail: sabrina.mueller@theol.uzh.ch https://doi.org/10.1515/zpt-2020-0058

Der englischsprachige Sammelband «Understanding Religion. Empirical Perspectives in Practical Theology» ist Hans-Georg Ziebertz gewidmet. Die Herausgeber verweisen in ihrer Widmung und Einleitung in den Band auf Ziebertz als einen der führenden Forscher, welcher die Empirische Theologie als methodisch fundierte und ernsthafte Form theologischer Reflexion etabliert hat. Dieser verstehe empirische Zugänge zu religiösen Phänomenen nicht als Antithese zur fundierten Theoriebildung, sondern als eigenständige Herangehensweise an die religiöse Praxis, die selbst zur Bildung neuer Theorien führt. Als Ziel des Sammelbandes wird genannt: «to honour Hans-Georg Ziebertz's academic portfolio by taking up his central interest in understanding religion from an international perspective» (6). Dazu werden Beiträge von wichtigen akademischen Begleiterinnen und Begleitern von Ziebertz veröffentlicht, die durch dessen Einsichten für ihre eigene religionsbezogene Forschung geprägt wurden. Der 254-seitige Sammelband selbst ist um die Kernthemen von Ziebertz' Forschungen herum strukturiert und besteht aus fünf Teilen. Nach einer Reflexion der Methodologie in Teil eins werden Forschungsprojekte und -ergebnisse zur Religiosität im zweiten Teil vorgestellt. Im dritten Teil wird ein besonderes Augenmerk auf das Thema Religion in der Gesellschaft gelegt und im vierten Teil auf Religion und Bildung eingegangen. Der fünfte Teil ist kurz gehalten und bietet einen möglichen Ausblick für die Zukunft Empirischer Theologie.

Der erste methodologische Teil besteht aus zwei Beiträgen, die von Friedrich Schweitzer und Alexander Unser verfasst wurden. Schweitzer plädiert in seinem Beitrag «Religious change in adolescence» für die Notwendigkeit von Langzeitstudien gerade im Themenhorizont von Religion. Denn Langzeitstudien können 
Erkenntnisse liefern, die bei einmaligen Erhebungen nicht verfügbar sind. So konnte zum Beispiel gezeigt werden, dass gute Erfahrungen während der Konfirmandenzeit ein Prädiktor für eine positive Einstellung gegenüber der Kirche in späteren Jahren sind. Der Artikel «on theology in empirical research» von Alexander Unser kann als gelungener Versuch gelesen werden, die erkenntnistheoretischen Überlegungen von Ziebertz fortzusetzen und zu einer Klärung des Verhältnisses zwischen Theologie und empirischer Forschung beizutragen. Dabei steht die Frage im Zentrum, ob empirische Methoden geeignet sind, die Aspekte der Wirklichkeit zu erforschen, auf die sich die Theologie bezieht: «whether and how a normative (theological) perspective on reality can be reconciled with empirical research» (32). Der Lösungsansatz wird darin gesehen, die Forschung hermeneutisch-theologisch zu rahmen.

Der zweite Teil des Sammelbandes besteht aus vier Beiträgen, die aktuelle Einblicke in Forschungsergebnisse zu gegenwärtig gelebter Religion geben. So wird von Anton A. Bucher die Stärke Empirischer Theologie darin gesehen, dass Stereotypen hinterfragt werden. Er erörtert in seinem Beitrag «There is more awe than expected in the $21^{\text {st }}$ century» die durchaus vorhandene Religiosität junger Menschen mithilfe eines psychologischen Konzeptes von Ehrfurcht. Dabei wird sichtbar, dass Ehrfurcht ein Gefühl ist, das integraler Bestandteil des Lebens ist und das sich je nach Alter und Geschlecht auf unterschiedliche Gegebenheiten beziehen kann. Im Beitrag von Leslie J. Francis und Ursula McKenna «The experience of victimisation among Christian adolescents in the UK» werden dagegen positive Faktoren von Religion für kirchlich aktive Jugendliche sowie deren Viktimisierung in einem primär säkularen Kontext diskutiert. Dabei wird ersichtlich, dass beispielsweise die Suizidalität durch religionsbezogenes Mobbing erhöht wird. Maria Klingenberg und Anders Sjöborg erörtern in ihrem Beitrag die methodologischen Herausforderungen, die religiöse Vielfalt, Individualisierung und Nicht-Religiosität für quantitative Studien zu zeitgenössischer Religiosität mit sich bringen. Der letzte Artikel in diesem Kapitel bildet eine finnische Perspektive von Kirsi Tirri und Elina Kuusisto ab. Die beiden Autorinnen wenden sich darin dem Thema Sinn zu: «What is purpose in life among Finish theology students?» Dabei stechen zwei Dinge als bedeutend heraus: zum einen verbinden die Studierenden Sinn mit «happiness» und Religion, zum anderen besteht ihr Ziel darin, ein glückliches Leben zu führen und Gottes Wille in ihrem Leben zu finden und zu verkünden.

Im dritten Teil des Sammelbandes steht das Thema Religion und Gesellschaft im Zentrum, dieses wird exemplarisch in fünf Artikeln dargestellt. Der erste Beitrag zu «Religion's impact on judical rights. An empirical study in the multireligious context of Indian democracy» von Francis-Vincent Anthony und Carl Sterkens hält als Fazit fest, dass persönliche Religiosität und religiöse Prägungen 
in Indien nur sehr begrenzten Einfluss auf die Rechtsprechung haben. Im zweiten Beitrag zu «Religion and humour - will the two sides ever meet?» stellt Pal Ketil Botvar das teileweise schwierige Verhältnis von Religion und Humor dar. Er führt in den Artikel ein, indem er den Anschlag von 2015 auf das Satiremagazin Charlie Hebdo thematisiert und danach fragt, ob Denominations- und Religionszugehörigkeit einen Einfluss darauf haben, wie mit religiösem Humor umgegangen werden kann. In seiner quantitativen Erhebung in Norwegen wird ersichtlich, dass dies durchaus der Fall ist. «Religious education in public perception» von Boris Kalbheim erhebt auf qualitative Weise Konzeptionen des Religionsunterrichts in der öffentlichen Wahrnehmung und dies durch eine Evaluation dessen, wie die Zeugnisnote im Fach Religion verstanden wird. Der vierte Beitrag, «The problem of defining religion in an increasingly individualized and functionally different society», von Per Pettersson erörtert die Schnittmenge zwischen theoretischen Definitionen des Religionsbegriffs, impliziten Definitionen durch empirisch Forschende und der Art und Weise, wie Religion im Kontext der Allgemeinen Erklärung der Menschenrechte zum Tragen kommt. Dabei zeigt sich, dass Religion ein facettenreiches Konzept ist und das Verständnis davon immer differenzierter wird. Den Abschluss dieses Kapitels bildet der Beitrag «Disaffiliation motives as indicators to better understand the relationship between religious institutions and individuals in modern Western society» von Ulrich Riegel und Tobias Faix. Riegel und Faix zeigen darin auf, dass die Konsumlogik gerade im Bereich Religion die Entfremdung von religiösen Institutionen vorantreibt. Dabei fördern folgende strukturelle Dimensionen die Entfremdung zur Kirche: die Performance der Kirche, die fehlende Beziehung zur Kirche und die mangelnde Glaubwürdigkeit der Kirche als Institution.

Im vierten Teil des Sammelbandes steht spezifisch das Thema religiöse Bildung im Fokus. In drei Beiträgen werden exemplarisch unterschiedliche Dimensionen empirischer Forschung in religiöser Bildung und Religionspädagogik veranschaulicht. Stefan Heil stellt in «Understanding religion professionally. The professional habitus of teachers in Catholic schools - a qualitative empirical study» einen qualitativen Forschungsansatz dar, bei dem Religion als Profession im Bildungskontext untersucht wird. Wolfram Weisse erörtert die Frage nach «Religious education in the context of pluralisation and secularisation» anhand von Fallstudien und Fragebögen. Dabei zeigt sich, dass die Vielfalt im Klassenzimmer im Allgemeinen und insbesondere im Hinblick auf die Lernmöglichkeiten aus einer Reihe von religiösen Gesichtspunkten begrüßt wird. Den Abschluss dieses Kapitels macht Joachim Willems mit seinem Artikel $\mathrm{zu}$ «Understanding religion and understanding concepts of religion. A challenge for (the theory of) religious education». Willems schlägt nach der Analyse von 30 Interviews mit nicht-religiösen, christlichen und muslimischen Jugendlichen vor, Religion als eine Dis- 
kurskategorie zu verstehen, gerade weil die Zugänge zur und das Verständnis von der Thematik erheblich variiert. Wird Religion als Diskurskategorie verstanden, kann dies für den Unterricht fruchtbar sein. Vor allem dann, wenn den Schülerinnen und Schülern die Unterschiede zwischen den verschiedenen Religionskonzepten bewusst gemacht werden. So können sie ihr Verständnis von Religion im Allgemeinen erweitern und unterschiedliche Sichtweisen bei der Definition von Religion kennenlernen bzw. sich damit auseinandersetzen.

Das letzte Kapitel Possible Futures besteht aus nur einem Beitrag von Johannes A. van der Ven mit dem Titel: «Three desiderata for Vatican III. An appraisal of Vatican II form the perspective of Human Rights». Besonders zu erwähnen ist bei diesem fünften Teil, dass der Beitrag unvollständig ist und dennoch so veröffentlich wurde, denn Johannes A. van der Ven, der zweite einflussreiche Begründer empirischer Theologie, erlag noch während des Verfassens dieses Beitrags seiner schweren Krankheit.

Der Sammelband ist ein Mosaik von quantitativen und qualitativen Zugängen und Reflexionen zum Phänomen Religion. Dennoch ist es den Herausgebern gelungen, mit den verschiedenen Diskurssträngen ein Ganzes zu kreieren. Understanding Religion ist logisch durchkomponiert und die doch sehr diversen Beiträge sind aufeinander bezogen, obwohl der plurale Zugriff auf Religion auffällt. Ganz unterschiedliche Perspektiven konnten eingeholt werden und verleihen dem Sammelband deshalb durchaus internationales Flair. Wer Understanding Religion mit der Erwartung liest, ein religionsphilosophisch-hermeneutisches Gesamtwerk in den Händen zu halten, wird enttäuscht sein. Denn Verständnisse von Religion werden tatsächlich und konsequent vom je untersuchten Phänomenbestand her quantitativ oder qualitativ konstruiert. Dieser Sammelband eignet sich für Leserinnen und Leser, die ein Interesse an empirischer Religionsforschung haben und ein Verständnis von Religion von dieser Perspektive aus konstruiert sehen wollen, oder sich über die Vielfalt und die Anwendungsmöglichkeiten empirischer Methoden im Bereich der Religionsforschung und nicht zuletzt auch in ihren Implikationen für weitere bildungstheoretische Diskurse zum Religionsverständnis informieren möchten. In diesem Horizont kann Understanding Religion als gelungener Diskurs zwischen Theologie und empirischer Religionsforschung angesehen werden. 